Check for updates

Cite this: Chem. Sci., 2019, 10, 145

๑ All publication charges for this article have been paid for by the Royal Society of Chemistry

\section{Aromatic secondary amine-functionalized fluorescent NO probes: improved detection sensitivity for NO and potential applications in cancer immunotherapy studies $\uparrow$}

\author{
Yingying Huo, $\S^{a}$ Junfeng Miao, $\S^{a}$ Junru Fang, ${ }^{a}$ Hu Shi, (D) Juanjuan Wang ${ }^{b}$ \\ and Wei Guo (iD *a
}

Tumor-associated macrophages (TAMs), constituting up to $50 \%$ of the solid tumor mass and commonly having a pro-tumoral M2 phenotype, are closely associated with decreased survival in patients. Based on the highly dynamic properties of macrophages, in recent years the repolarization of TAMs from protumoral M2 phenotype to anti-tumoral M1 phenotype by various strategies has emerged as a promising cancer immunotherapy approach for improving cancer therapy. Herein, we present an aromatic secondary amine-functionalized Bodipy dye 1 and its mitochondria-targetable derivative Mito1 as fluorescent NO probes for discriminating M1 macrophages from M2 macrophages in terms of their difference in inducible NO synthase (iNOS) levels. The two probes possess the unique ability to simultaneously respond to two secondary oxides of $\mathrm{NO}$, i.e., $\mathrm{N}_{2} \mathrm{O}_{3}$ and $\mathrm{ONOO}^{-}$, thus being more sensitive and reliable for reflecting intracellular $\mathrm{NO}$ than most of the existing fluorescent $\mathrm{NO}$ probes that usually respond to $\mathrm{N}_{2} \mathrm{O}_{3}$ only. With 1 as a representative, the discrimination between $\mathrm{M} 1$ and $\mathrm{M} 2$ macrophages, evaluation of the repolarization of TAMs from pro-tumoral M2 phenotype to anti-tumoral M1 phenotype, and visualization of NO communication during the immune-mediated phagocytosis of cancer cells by M1 macrophages have been realized. These results indicate that our probes should hold great potential for imaging applications in cancer immunotherapy studies and relevant anti-cancer drug screening.
Received 19th August 2018

DOI: $10.1039 / \mathrm{c} 8 \mathrm{sc} 03694 \mathrm{~b}$

rsc.li/chemical-science

\section{Introduction}

Macrophages are specialized immune cells found all over the body that exist primarily to engulf and digest cellular debris, foreign substances, microbes, and cancer cells in a process called phagocytosis. Macrophages are particularly active in inflammation and infection, under which conditions, blood monocytes are recruited into the tissue where they differentiate into macrophages. ${ }^{1}$ Notably, macrophages are the most wellcharacterized type of tumor-infiltrating immune cells, and play crucial roles from anti-tumor to tumor progression and metastasis. Accumulating evidence reveals that the dual functions of macrophages can be attributed to their ability to adapt to the macroenvironment that leads to two main polarized

${ }^{a}$ School of Chemistry and Chemical Engineering, Shanxi University, Taiyuan 030006, China. E-mail: guow@sxu.edu.cn

${ }^{b}$ Scientific Instrument Center, Shanxi University, Taiyuan 030006, China

$\dagger$ Dedicated to Prof. Jin-Pei Cheng on the occasion of his 70th birthday.

\$ Electronic supplementary information (ESI) available: Synthesis, experimental procedures, supplemental spectra and imaging data, and ${ }^{1} \mathrm{H}-,{ }^{13} \mathrm{C}-\mathrm{NMR}$, and MS spectra. See DOI: $10.1039 / \mathrm{c} 8 \mathrm{sc} 03694 \mathrm{~b}$

$\S$ These authors contributed equally to this work. phenotypes, i.e., classically activated M1 macrophages and alternatively activated M2 macrophages. ${ }^{2-4}$ M1 macrophages, characterized by the expression of high-level inducible nitric oxide (NO) synthase (iNOS) as well as some pro-inflammatory cytokines, such as IL-12, ${ }^{5-7}$ have high bactericidal and tumoricidal activity partially due to their ability to secrete high levels of reactive oxygen/nitrogen species (ROS/RNS), such as hydrogen peroxide $\left(\mathrm{H}_{2} \mathrm{O}_{2}\right)$, superoxide $\left(\mathrm{O}_{2}^{-\cdot}\right)$, and $\mathrm{NO}$ and its secondary metabolites dinitrogen trioxide $\left(\mathrm{N}_{2} \mathrm{O}_{3}\right)$ and peroxynitrite $\left(\mathrm{ONOO}^{-}\right)$. In contrast, $\mathrm{M} 2$ macrophages, characterized by the expression of arginase 1 (Arg-1) and anti-inflammatory cytokines, such as IL-10, commonly have a low level of iNOS $^{5-7}$ and can assist tumor development by inducing angiogenesis, remodeling the extracellular matrix, stimulating cancer cell proliferation, and inhibiting adaptive immunity. In fact, under cancer-initiating conditions, the infiltrated macrophages have an M1 phenotype and are anti-tumoral; however, their continued presence in a tumor microenvironment polarizes them to tumor-associated macrophages (TAMs), which commonly have an M2 phenotype and are closely associated with decreased survival in patients due to their pro-tumoral role. ${ }^{2-4}$ Of note, the polarization of macrophages is a highly 
dynamic process and the phenotype of M1- or M2-polarized macrophages can be reversed depending on the microenvironmental cues they receive. ${ }^{8}$ For instance, the reversion of macrophages from M2 phenotype to M1 phenotype and reduction of immunosuppressive effects from the M2 population have been observed when TAMs were treated with interferon- $\gamma /$ lipopolysaccharide (IFN- $\gamma /$ LPS); ${ }^{\mathbf{9}, 10}$ in patients with extended survival, the M1 macrophages account for the majority of macrophages present within tumors, ${ }^{\mathbf{1 1}}$ distinct from the cases in tumor development and metastasis, where macrophages predominantly exhibit a pro-tumoral M2 phenotype. ${ }^{2-7}$ Based on these discoveries, the repolarization of TAMs from M2 phenotype to M1 phenotype to activate their anti-tumoral potential by various strategies has emerged as an attractive and promising approach in cancer immunotherapy in recent years. ${ }^{2,12-17}$ In this context, the development of efficient methods that can discriminate M1 macrophages from M2 macrophages is of crucial guiding significance for cancer immunotherapy studies and relevant anti-cancer drug screening.

Although immunohistological quantification, enzyme linked immunosorbent assay (ELISA), and Western blot analysis of various biomarkers have routinely been used to distinguish between M1 and M2 macrophages, ${ }^{5-7,17}$ these methods are complex, time-consuming, and especially incompatible with living systems. By comparison, fluorescent probe-based techniques, which have become the gold standard for detection and imaging of various biological species in living systems, are the most promising to overcome these limitations due to their simplicity, convenience, sensitivity, noninvasiveness, and realtime spatial imaging capacity ${ }^{18,19}$ However, the attractive techniques have never been exploited to identify M1 or M2 macrophages to date. Given that M1 macrophages express higher levels of iNOS than M2 macrophages, we envisioned that fluorescent NO probes when properly designed should have the potential to distinguish between M1 and M2 macrophages in terms of their difference in the iNOS level and thus the NO level.

Among various fluorescent NO probes, ${ }^{20-28} \mathrm{o}$-diamine-based ones, pioneered by Nagano's group, are by far the most often studied and applied fluorescent NO probes. ${ }^{23-28}$ The corresponding sensing mechanism is based on the reaction of the $o$ diamine group with the autoxidation product of NO, i.e., dinitrogen trioxide $\left(\mathrm{N}_{2} \mathrm{O}_{3}\right)$, ${ }^{29}$ to form the benzotriazole derivative, thereby triggering a fluorescence off-on response by inhibiting the photoinduced electron transfer (РеT) process. Although fluorescent NO probes of this kind have widely been applied in biological systems, some limitations still remain, such as possible interference by dehydroascorbic acid (DHA)/ascorbic acid (AA)/methylglyoxal (MGO) $)^{30-33}$ and a relatively long response time (commonly more than $5 \mathrm{~min}$ ). To overcome these limitations, in recent years some new strategies have been actively developed, such as diazo ring formation, ${ }^{34,35}$ reductive deamination, ${ }^{36,37}$ monoprotection of vicinal diamine groups, ${ }^{38-41}$ aromatization of Hantzsch ester, ${ }^{\mathbf{4 2 , 4 3}}$ and $\mathrm{N}$-nitrosation of aromatic secondary amines. ${ }^{\mathbf{4 4 - 4 7}}$ Yet despite the remarkable progress that has already been achieved, a widely overlooked issue is that almost all of these probes, including 0 diamine-based ones, can reflect intracellular NO only by reacting with its autooxidation product $\mathrm{N}_{2} \mathrm{O}_{3}$, which would inevitably decrease the detection sensitivity for $\mathrm{NO}$ given that NO could also rapidly react with $\mathrm{O}_{2}^{-}$to generate $\mathrm{ONOO}^{-}$at near diffusion control $\left(\sim 10^{10} \mathrm{M}^{-1} \mathrm{~S}^{-1}\right),{ }^{48}$ and that these probes usually fail to give a fluorescence response toward $\mathrm{ONOO}^{-}$. The situation may be especially serious during the immune response of macrophages, where large amounts of $\mathrm{NO}$ and $\mathrm{O}_{2}{ }^{-}$. were simultaneously produced and coexisted with $\mathrm{O}_{2} \cdot{ }^{49}$ Thus, the development of new fluorescent NO probes that can sensitively sense both $\mathrm{N}_{2} \mathrm{O}_{3}$ and $\mathrm{ONOO}^{-}$is highly desired for improving not only the detection sensitivity of NO but also the reliability in distinguishing between M1 and M2 macrophages.

Recently, we reported for the first time that aromatic secondary amines could function as both the reaction group and PeT donor to construct fluorescent NO probes. ${ }^{44}$ However, like most of the previous reports, the as-obtained probe, i.e., $\mathrm{N}$ benzyl-4-hydroxyaniline-functionalized Bodipy, only exhibited a selective fluorescence off-on response toward $\mathrm{N}_{2} \mathrm{O}_{3}$ but not $\mathrm{ONOO}^{-}$. Further studies revealed that although not giving a fluorescence response, the probe could react with $\mathrm{ONOO}^{-}$to lead to a nonfluorescent debenzylation product. This means that in practical bioimaging assays, the probe would probably suffer from the risk of being consumed by coexisting $\mathrm{ONOO}^{-}$, thereby resulting in decreased sensitivity for NO. However, to our delight, when $N$-benzyl-4-methoxyaniline was employed as the reaction group instead of the $N$-benzyl-4-hydroxyaniline group mentioned above, the newly developed fluorescent probe, i.e., $N$-benzyl-4-methoxyaniline-functionalized Bodipy 1 and its mitochondria-targetable derivative Mito1 (Scheme 1), not only overcame the shortcomings of classic $o$-diamine-type probes, such as possible interference by DHA/AA/MGO and a long response time, but also displayed a significant fluorescence off-on response for both $\mathrm{N}_{2} \mathrm{O}_{3}$ and $\mathrm{ONOO}^{-}$. The unique sensing properties endow the probes with high sensitivity for reflecting intracellular NO as indicated by their ability to image basal and endogenous NO in living cells. With 1 as

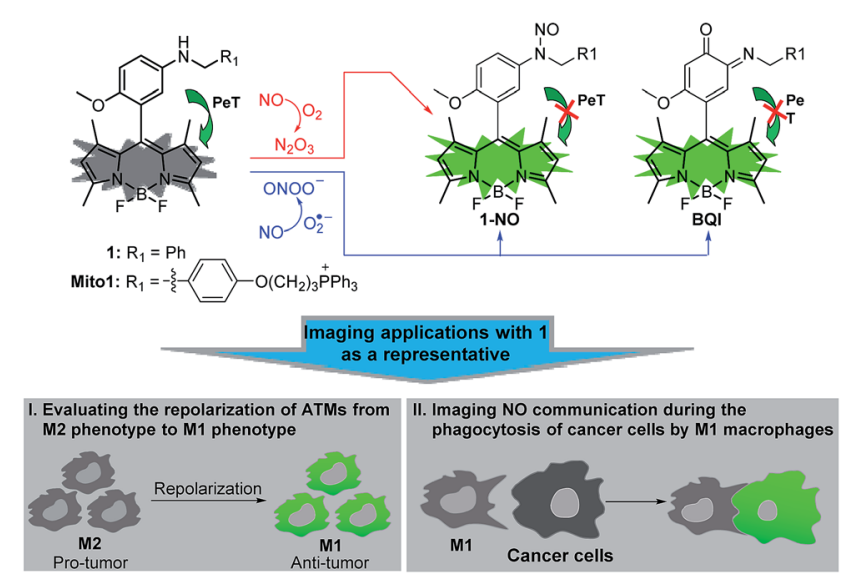

Scheme 1 Proposed fluorescence sensing mechanisms of $1 /$ Mito 1 for $\mathrm{N}_{2} \mathrm{O}_{3}$ and $\mathrm{ONOO}^{-}$and potential imaging applications of 1 as a representative in evaluating the repolarization of TAMs from M2 phenotype to M1 phenotype and imaging NO communication during the phagocytosis of cancer cells by M1 macrophages. 
a representative, we have successfully realized the discrimination between M1 and M2 macrophages and visualization of the repolarization of TAMs from M2 phenotype to M1 phenotype induced by IFN- $\gamma /$ LPS. Also, we confirmed that during the immune-mediated phagocytosis of cancer cells by M1 macrophages, NO secreted by M1 macrophages could diffuse across the cancer cell membrane to exert its tumoricidal action by producing cytotoxic $\mathrm{N}_{2} \mathrm{O}_{3}$ and $\mathrm{ONOO}^{-}$. These findings strongly indicate that our probes should hold great potential for imaging applications in cancer immunotherapy studies and relevant drug screening.

\section{Results and discussion}

\section{Synthesis and spectral response of 1 and Mito1 for $\mathrm{N}_{2} \mathrm{O}_{3}$ and $\mathrm{ONOO}^{-}$}

Probes 1 and Mito1 could be easily synthesized by a simple three-step procedure starting from commercially available 2methoxy-5-nitrobenzaldehyde, including the initial synthesis of Bodipy dye, subsequent reduction of the nitro group to the amino group, and final reductive amination with corresponding benzaldehydes. The detailed synthesis and characterization data of 1 and Mito1 are presented in the ESI.\$ With the two probes in hand, we first evaluated the spectral response of $\mathbf{1}$ for $\mathrm{N}_{2} \mathrm{O}_{3}$ and $\mathrm{ONOO}^{-}$in PBS buffer $(50 \mathrm{mM}, \mathrm{pH}$ 7.4, containing $20 \% \mathrm{CH}_{3} \mathrm{CN}$ ). As shown in Fig. $1 \mathrm{~A}$, the solution of 1 itself had an extremely poor fluorescence at $518 \mathrm{~nm}(\Phi=0.016)$, presumably
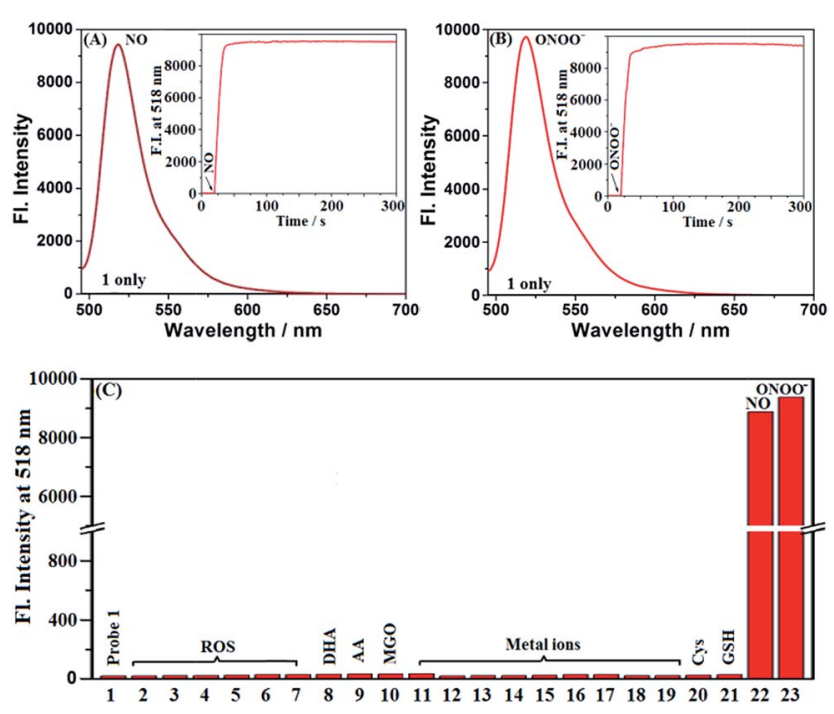

Fig. 1 ( $\mathrm{A}$ and $\mathrm{B})$ Fluorescence spectra of $1(4 \mu \mathrm{M})$ treated with and without $\mathrm{NO}(50 \mu \mathrm{M})$ or $\mathrm{ONOO}^{-}(20 \mu \mathrm{M})$ under aerobic conditions. Inset: the time-dependent fluorescence intensity changes of $1(4 \mu \mathrm{M})$ treated with and without $\mathrm{NO}$ or $\mathrm{ONOO}^{-}(20 \mu \mathrm{M})$. (C) Fluorescence intensities of $1(4 \mu \mathrm{M})$ treated with various competitive species at the time point of 2 min. (1) 1 only; (2) $\mathrm{ClO}^{-}$; (3) $\mathrm{H}_{2} \mathrm{O}_{2}$; (4) $\mathrm{O}_{2}{ }^{--}$; (5) ${ }^{1} \mathrm{O}_{2}$; (6)

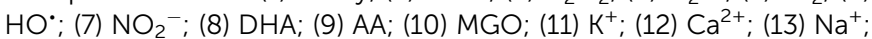
(14) $\mathrm{Mg}^{2+} ;$ (15) $\mathrm{Al}^{3+} ;$ (16) $\mathrm{Zn}^{2+} ;$ (17) $\mathrm{Fe}^{2+} ;$ (18) $\mathrm{Fe}^{3+} ;$ (19) $\mathrm{Cu}^{2+} ;$ (20) Cys; (21) $\mathrm{GSH}$; (22) NO; (23) $\mathrm{ONOO}^{-}$. Concentrations for (2-7), $100 \mu \mathrm{M}$; for (8-21), $1 \mathrm{mM}$; for (22), $50 \mu \mathrm{M}$; for (23), $20 \mu \mathrm{M}$. Conditions: PBS (50 mM, $\mathrm{pH} 7.4$, containing $20 \% \mathrm{CH}_{3} \mathrm{CN}$ ); $\lambda_{\mathrm{ex}}=485 \mathrm{~nm} ; \lambda_{\mathrm{em}}=518 \mathrm{~nm}$; slits: $5 /$ $10 \mathrm{~nm}$; voltage: $600 \mathrm{~V}$. due to PeT from the electron-rich $N$-benzyl-4-methoxyaniline unit to the excited Bodipy core; however, upon treatment with excess NO solution under aerobic conditions (conditions for producing $\left.\mathrm{N}_{2} \mathrm{O}_{3}\right),{ }^{23-28}$ a significant fluorescence enhancement (880-fold) was observed from the dark background, indicating that the reaction of 1 with $\mathrm{N}_{2} \mathrm{O}_{3}$ could efficiently block the PeT process and thereby turn-on the fluorescence. Notably, as revealed by the kinetics study (Fig. 1A, inset), the fluorescence response of $\mathbf{1}$ for $\mathrm{N}_{2} \mathrm{O}_{3}$ was fairly fast and could be completed within $10 \mathrm{~s}$, indicative of the potential of $\mathbf{1}$ for real-time imaging of endogenous $\mathrm{N}_{2} \mathrm{O}_{3}$ in biosystems. The fluorescence titration assay was further performed to evaluate the sensitivity of $\mathbf{1}$ for $\mathrm{N}_{2} \mathrm{O}_{3}$. As shown in Fig. S1 (ESI:), a good linearity between the fluorescence intensities at $518 \mathrm{~nm}$ and the concentrations of added $\mathrm{NO}(0-16 \mu \mathrm{M})$ was observed, and the detection limit (DL) for $\mathrm{N}_{2} \mathrm{O}_{3}\left(4 \mathrm{NO}+\mathrm{O}_{2}=2 \mathrm{~N}_{2} \mathrm{O}_{3}\right)$ was calculated to be as low as $0.4 \mathrm{nM}$ based on $3 \sigma / k$. When compared with the $N$-benzyl-4hydroxyaniline-functionalized Bodipy probe reported by us previously, ${ }^{44} 1$ displays a bigger fluorescence off-on response and higher detection sensitivity for $\mathrm{N}_{2} \mathrm{O}_{3}$, indicating that the $N$ benzyl-4-methoxyaniline group of $\mathbf{1}$ should be a more excellent reaction group for $\mathrm{N}_{2} \mathrm{O}_{3}$ than the $N$-benzyl-4-hydroxyaniline group. Importantly, when 1 was treated with excess $\mathrm{ONOO}^{-}$ under the same conditions, a rapid and great fluorescence offon response was also observed, which is almost consistent with the case of $\mathrm{N}_{2} \mathrm{O}_{3}$ in either fluorescence intensity or response kinetics (Fig. 1B). Moreover, a good linear correlation between the fluorescence intensities and the concentrations of $\mathrm{ONOO}^{-}$ in the range of $0-2.5 \mu \mathrm{M}$ was also found (Fig. S2, ESIt), and the $\mathrm{DL}$ for $\mathrm{ONOO}^{-}$was calculated to be $0.14 \mathrm{nM}$ based on $3 \sigma / k$. The results reveal that 1 is extremely sensitive not only for $\mathrm{N}_{2} \mathrm{O}_{3}$ but also for $\mathrm{ONOO}^{-}$, thus being very promising as a more sensitive indicator to reflect intracellular NO.

To establish the selectivity, we tested the fluorescence response of 1 toward various biologically relevant species, including reactive oxygen/nitrogen species (ROS/RNS: $\mathrm{ClO}^{-}$, $\mathrm{H}_{2} \mathrm{O}_{2}, \mathrm{O}_{2}{ }^{--},{ }^{1} \mathrm{O}_{2}, \cdot \mathrm{OH}, \mathrm{NO}_{2}{ }^{-}$, NO, and $\mathrm{ONOO}^{-}$), DHA/AA/MGO, metal ions $\left(\mathrm{K}^{+}, \mathrm{Ca}^{2+}, \mathrm{Na}^{+}, \mathrm{Mg}^{2+}, \mathrm{Al}^{3+}, \mathrm{Zn}^{2+}, \mathrm{Fe}^{2+}, \mathrm{Fe}^{3+}\right.$, and $\mathrm{Cu}^{2+}$ ), and biothiols (Cys and GSH). As shown in Fig. 1C, the treatment of 1 with either $\mathrm{N}_{2} \mathrm{O}_{3}\left(\mathrm{NO} / \mathrm{O}_{2}\right)$ or $\mathrm{ONOO}^{-}$could induce a significant fluorescence off-on response, while the other competitive species failed to give any obvious fluorescence alteration of $\mathbf{1}$, indicating that the probe is highly specific for $\mathrm{N}_{2} \mathrm{O}_{3}$ and $\mathrm{ONOO}^{-}$. In addition, 1 was almost nonfluorescent in the $\mathrm{pH}$ range of $5-9$, but displayed the best fluorescence response for $\mathrm{N}_{2} \mathrm{O}_{3}$ and $\mathrm{ONOO}^{-}$at 7.4 (Fig. S3, ESI:), thus being suitable for imaging application at physiological $\mathrm{pH}$.

Encouraged by the above results, we further tested the fluorescence sensing performances of Mito1 for both $\mathrm{N}_{2} \mathrm{O}_{3}$ and $\mathrm{ONOO}^{-}$under the same conditions. Indeed, the probe was designed as a mitochondria-targetable fluorescent NO probe by installing a mitochondria-targeted triphenylphosphonium (TPP) cation ${ }^{50,51}$ to the molecular skeleton of 1 . Interestingly, as shown in Fig. S4-8 (ESI), Mito1 displayed almost the same sensing performances for $\mathrm{N}_{2} \mathrm{O}_{3}$ and $\mathrm{ONOO}^{-}$as $\mathbf{1}$, such as the significant and rapid fluorescence off-on response, high selectivity and sensitivity, and excellent fluorescence response at 
physiological $\mathrm{pH}$, indicating that the TPP cation rarely affects the sensing performances of Mito1 toward $\mathrm{N}_{2} \mathrm{O}_{3}$ and $\mathrm{ONOO}^{-}$. Thus, Mito1 should have the potential to sensitively and specifically reflect mitochondrial NO.

Overall, as revealed by the above assays, 1 and Mito1 displayed high sensitivity, excellent selectivity, and fast response ability for both $\mathrm{N}_{2} \mathrm{O}_{3}$ and $\mathrm{ONOO}^{-}$under the simulated physiological conditions, thus holding great potential for probing NO-related physiology and pathology.

\section{Sensing mechanisms of 1 and Mito1 for $\mathrm{N}_{2} \mathrm{O}_{3}$ and $\mathrm{ONOO}^{-}$}

With 1 as a representative, we subsequently studied the sensing mechanisms of the probe for both $\mathrm{N}_{2} \mathrm{O}_{3}$ and $\mathrm{ONOO}^{-}$by HPLCHRMS assays. As shown in Fig. S9 (ESI), the HPLC analysis showed that the reaction of 1 with $\mathrm{N}_{2} \mathrm{O}_{3}$ mainly produced a new peak, which could be assigned to N-nitroso product 1-NO in terms of HRMS data $\left(\mathrm{m} / \mathrm{z}\right.$ calcd for $\left[\mathrm{M}+\mathrm{H}^{+}\right] 489.2273$, found 489.2263) (Scheme 2A). This is consistent with the previous report that aromatic secondary amines can react with NO under aerobic conditions to give the N-nitroso product. ${ }^{\mathbf{4 4}}$ However, in the case of $\mathrm{ONOO}^{-}$, in addition to the major $\mathrm{N}$-nitroso product 1-NO $\left(m / z\right.$ : calcd for $\left[\mathrm{M}+\mathrm{H}^{+}\right] 489.2273$, found 489.2263), two unknown new products, one major and the other minor, were observed as well in HPLC analysis (Fig. S10, ESI†). Considering that secondary amines can react with $\mathrm{ONOO}^{-}$to produce both $\mathrm{N}$-nitroso and N-nitro products, ${ }^{52}$ we proposed a possible reaction mechanism as follows (Scheme $2 \mathrm{~B}$ ): first, the reaction of 1 with $\mathrm{ONOO}^{-}$produced the $\mathrm{N}$-nitroso product 1-NO and N-nitro product 1- $\mathrm{NO}_{2}$; due to the strong push-pull electronic interaction between the $-\mathrm{OMe}$ group and $-\mathrm{NO}_{2}$ group, 1- $\mathrm{NO}_{2}$ was unstable and underwent an intramolecular two-electron transfer to give $p$-benzoquinone imine intermediate $\mathbf{B 1}$; the intermediate was also unstable and could be attached by the $\mathrm{H}_{2} \mathrm{O}$ molecule via a Michael addition-like reaction to generate intermediate $\mathbf{B} 2$; the oxidative dehydrogenation of $\mathbf{B} 2$ afforded $o$-benzoquinone imine BQI as the final product of the reaction
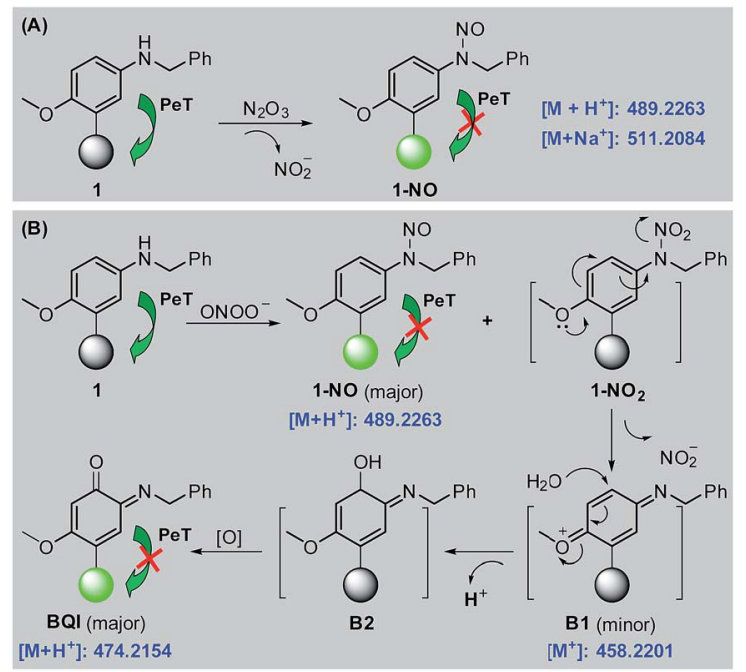

Scheme 2 Proposed sensing mechanisms of 1 for $\mathrm{N}_{2} \mathrm{O}_{3}(A)$ and $\mathrm{ONOO}^{-}$(B), respectively. Round balls represent the Bodipy core. pathway. According to the proposed mechanism, the abovementioned two unknown products could reasonably be assigned to B1 (minor) and BQI (major) in terms of the excellent matching of calculated and observed $\mathrm{m} / \mathrm{z}$ values (for B1, calcd for $\left[\mathrm{M}^{+}\right]$458.2215, found 458.2201; for BQI, calcd for $\left[\mathrm{M}+\mathrm{H}^{+}\right]$ 474.2164, found 474.2154) (Fig. S10, ESI\$). Thus, the HPLCHRMS assays nicely support our proposed reaction mechanisms of 1 for $\mathrm{N}_{2} \mathrm{O}_{3}$ and $\mathrm{ONOO}^{-}$.

Further, the fluorescence off-on response of $\mathbf{1}$ for $\mathrm{N}_{2} \mathrm{O}_{3}$ and $\mathrm{ONOO}^{-}$by inhibiting the PeT process was rationalized by the Frontier orbital energy diagrams of 1, 1-NO, and BQI, obtained by Becke's three-parameter hybrid exchange function with the Lee-Yang-Parr gradient-corrected correlation functional (B3LYP functional) and $6-31+\mathrm{G}^{*}$ basis set (Fig. S11, ESI + ). To support the conclusion, we studied the fluorescence changes of $\mathbf{1}$ in mixed water-glycerol systems (0-100\% of glycerol) with varied viscosity. As shown in Fig. S12 (ESI ), in these cases 1 still displayed negligible fluorescence, strongly indicating that no fluorescence of $\mathbf{1}$ is indeed due to the PeT process, rather than rotation or vibration-relevant nonradiative processes. ${ }^{53}$

\section{Basic imaging ability of 1 and Mito1 for $\mathrm{N}_{2} \mathrm{O}_{3}$ and $\mathrm{ONOO}^{-}$in living cells as well as their subcellular distribution}

Prior to biological imaging applications, the cytotoxicity of $\mathbf{1}$ and Mito1 was first tested in HeLa cells by MTT assays. As shown in Fig. S13 (ESI+), after $24 \mathrm{~h}$ of cellular internalization of less than $8 \mu \mathrm{M}$ of $\mathbf{1}$ or Mito1, $>90 \%$ of the cells remained viable, indicative of the good biocompatibility of the two probes. Notably, 1 displayed an obviously lower cytotoxicity than Mito1, presumably due to its uncharged property reducing its interaction with either the negatively charged DNA or the mitochondrial membrane with highly negative potential. Even so, in order to reduce the interference to cell proliferation and physiology, a low concentration of 1 or Mito1 $(2 \mu \mathrm{M})$, survival rates close to $100 \%$ in the case, was used in the subsequent bioimaging assays. Subsequently, we evaluated the selectivity of 1 or Mito1 for $\mathrm{N}_{2} \mathrm{O}_{3}$ and $\mathrm{ONOO}^{-}$in human cervical cancer HeLa cells. As shown in Fig. 2, HeLa cells loaded with 1 or Mito1 showed negligible background fluorescence; when the 1- or Mito1-loaded HeLa cells were treated with NOC-9 (a commercial NO donor) or SIN-1 (a commercial ONOO $^{-}$donor), a strong intracellular green fluorescence was observed for both cases; when 1- or Mito1-loaded HeLa cells were treated with representative ROS, such as $\mathrm{H}_{2} \mathrm{O}_{2}$ and $\mathrm{ClO}^{-}$, almost no any intracellular green fluorescence was found. The results suggest that 1 and Mito1 still possess high specificity for $\mathrm{N}_{2} \mathrm{O}_{3}$ and $\mathrm{ONOO}^{-}$in a cell environment.

Encouraged by the above results, we further tested the ability of 1 or Mito1 for imaging endogenous $\mathrm{N}_{2} \mathrm{O}_{3}$ and $\mathrm{ONOO}^{-}$in mouse RAW264.7 macrophages that are known to express highlevel iNOS upon stimulation by LPS/IFN- $\gamma .{ }^{54}$ As shown in Fig. 3, RAW264.7 cells themselves were nonfluorescent; upon incubation with 1 or Mito1, the cells displayed a weak yet clear intracellular green fluorescence; when the cells were pretreated with NO synthase inhibitor aminoguanidine $(\mathrm{AG})^{55}$ and then treated with 1 or Mito1, the intracellular green fluorescence was greatly 

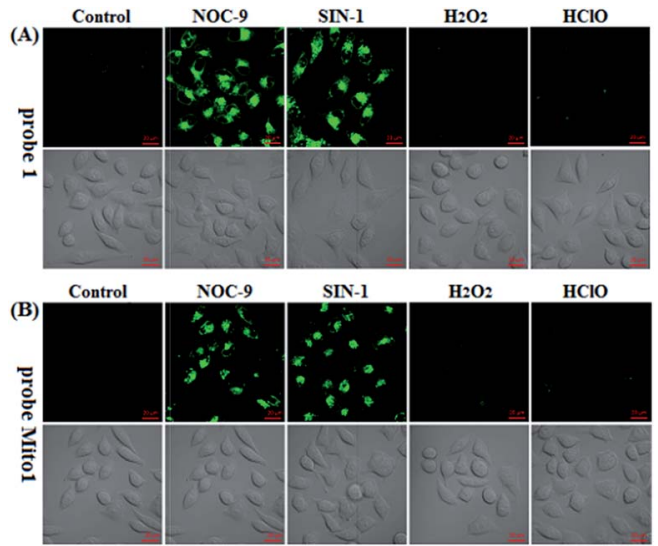

Fig. 2 Confocal images of HeLa cells pretreated with $1(2 \mu \mathrm{M})(\mathrm{A})$ or Mito1 ( $2 \mu \mathrm{M})(\mathrm{B})$ for $20 \mathrm{~min}$, and then treated with NOC-9 $(25 \mu \mathrm{M})$, SIN$1(10 \mu \mathrm{M}), \mathrm{H}_{2} \mathrm{O}_{2}(50 \mu \mathrm{M})$, and $\mathrm{ClO}^{-}(50 \mu \mathrm{M})$ for 20 min, respectively, in PBS. Emission was collected at $493-600 \mathrm{~nm}\left(\lambda_{\mathrm{ex}}=488 \mathrm{~nm}\right)$. Scale bar: $20 \mu \mathrm{m}$.
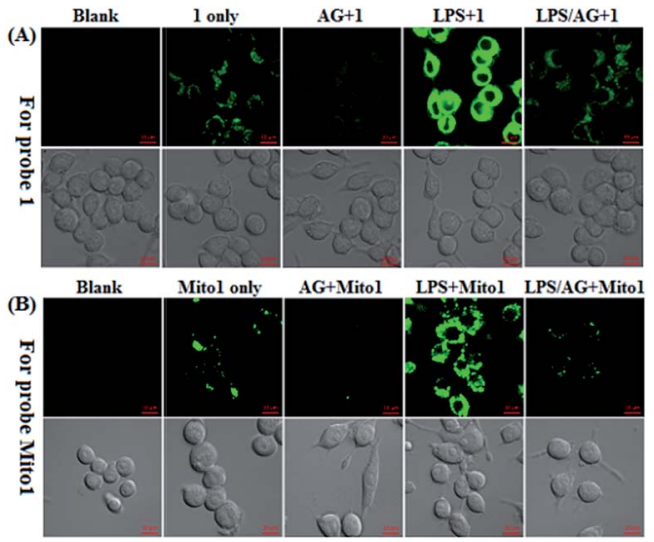

Fig. 3 Confocal images of the basic and stimulator-induced $\mathrm{NO}$ in RAW 264.7 macrophages using 1 (A) and Mito1 (B). For imaging of intracellular basal NO, cells were treated directly with 1 or Mito1 $(2 \mu \mathrm{M}$, $20 \mathrm{~min}$ ) in PBS; for imaging of the stimulator-induced NO, cells were pretreated with stimulators LPS $\left(20 \mu \mathrm{g} \mathrm{mL}^{-1}\right) / \mathrm{INF}-\gamma(150$ units per $\mathrm{mL})$ for $6 \mathrm{~h}$ in PBS and then treated with 1 or Mito1 ( $2 \mu \mathrm{M}, 20 \mathrm{~min})$; for inhibition assays, cells were pretreated with LPS $\left(20 \mu \mathrm{g} \mathrm{mL}^{-1}\right) / \mathrm{INF}-\gamma$ (150 units per $\mathrm{mL}$ ) for $6 \mathrm{~h}$ in the presence of $A G(0.5 \mathrm{mM})$ and then treated with 1 or Mito1 ( $2 \mu \mathrm{M}, 20$ min). Emission was collected at 493$600 \mathrm{~nm}\left(\lambda_{\mathrm{ex}}=488 \mathrm{~nm}\right)$. Scale bar: $10 \mu \mathrm{m}$.

inhibited. The results indicate that the two probes are sensitive enough to determine the basal level of intracellular NO by responding to $\mathrm{N}_{2} \mathrm{O}_{3}$ or $\mathrm{ONOO}^{-}$. Further, when the cells were stimulated with LPS/IFN- $\gamma$ and then treated with 1 or Mito1, a bright intracellular green fluorescence was clearly observed; when the cells were stimulated with LPS/IFN- $\gamma$ in the presence of AG and then treated with $\mathbf{1}$ or Mito1, the intracellular green fluorescence was greatly inhibited. Thus, the two probes can be used to sense the LPS/IFN- $\gamma$-triggered outburst of endogenous NO, indicating their potential for studying various NO-related pathophysiological events.

Also, we tested the subcellular distribution of $\mathbf{1}$ and Mito1 in HeLa cells by costaining assays. In the assays, NOC-9 was used to light up the two probes in cells, and Pearson's correlation coefficient $(R)$ was used to analyze the linear correlation of fluorescence signals between the green channel (for probes) and red channel (for commercial trackers). As shown in Fig. 4A, when HeLa cells were co-incubated with 1/MitoTracker or 1/LysoTracker followed by NOC-9 treatment, a poor overlapping image was observed for both cases $(R=0.35$ and 0.31 , respectively), indicating that $\mathbf{1}$ is not specific for either mitochondria or lysosomes. However, when HeLa cells were co-incubated with 1, MitoTracker, and LysoTracker followed by NOC-9 treatment, we observed an excellent overlapping image from the green channel and red channel $(R=0.88)$, indicating that 1 was indeed distributed over both mitochondria and lysosomes. However, as shown in Fig. 4B, when HeLa cells were coincubated with Mito1/MitoTracker or Mito1/LysoTracker followed by NOC-9 treatment, a good overlapping image along with a high Pearson's correlation coefficient was only observed for the former $(R=0.89)$ but not the latter $(R=0.10)$, indicating that Mito1 could preferably localize in mitochondria rather than lysosomes. The excellent localization of Mito1 in mitochondria could indeed be attributed to its lipophilic TPP cation that directs the probe into mitochondria by the highly negative potential of the mitochondrial membrane (about $-180 \mathrm{mV}$ ). ${ }^{50,51}$

\section{Potential applications in cancer immunotherapy studies}

Having established their excellent imaging ability for $\mathrm{NO}$ in chemical systems and living cells by responding to both $\mathrm{N}_{2} \mathrm{O}_{3}$ and $\mathrm{ONOO}^{-}$, we envisioned that $\mathbf{1}$ and Mito1 should have the

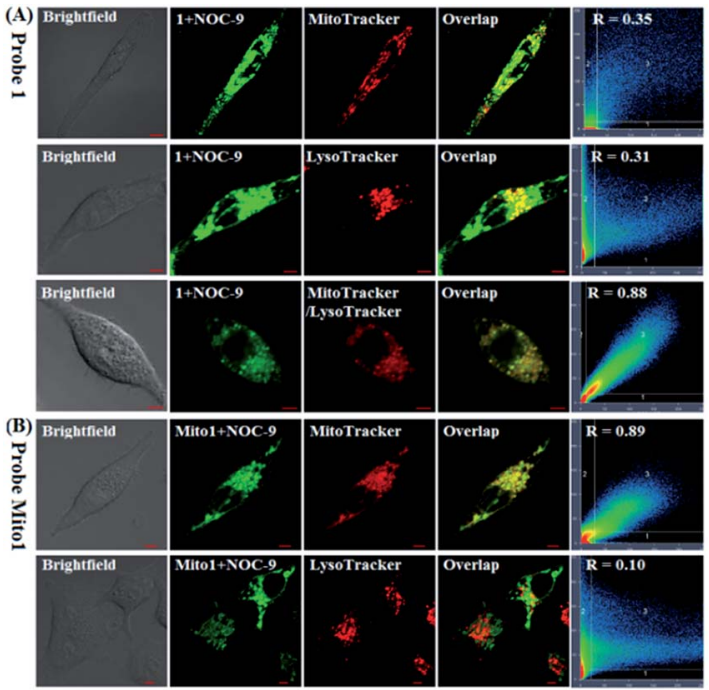

Fig. 4 (A) Confocal images of HeLa cells co-stained with $1(2 \mu \mathrm{M}) /$ MitoTracker Red FM (0.3 $\mu \mathrm{M}), 1(2 \mu \mathrm{M}) /$ LysoTracker Deep Red (0.07 $\mu \mathrm{M})$, and $1(2 \mu \mathrm{M}) /$ MitoTracker Red FM $(0.3 \mu \mathrm{M}) /$ LysoTracker Deep Red $(0.07 \mu \mathrm{M})$ for $30 \mathrm{~min}$, respectively, in PBS, and then treated with NOC$9(30 \mu \mathrm{M}, 20 \mathrm{~min})$. (B) Confocal images of HeLa cells co-stained with Mito1 $(2 \mu \mathrm{M})$ /MitoTracker Red FM $(0.3 \mu \mathrm{M})$ and Mito1 $(2 \mu \mathrm{M}) / \mathrm{Lyso-}$ Tracker Deep Red $(0.07 \mu \mathrm{M})$ for 30 min, respectively, in PBS, and then treated with NOC-9 (25 $\mu$ M, 20 min). For 1 and Mito1, emission was collected at 493-600 nm ( $\lambda_{\text {ex }}=488 \mathrm{~nm}$ ); for MitoTracker and LysoTracker, emission was collected at $638-747 \mathrm{~nm}\left(\lambda_{\mathrm{ex}}=633 \mathrm{~nm}\right)$. Scale bar: $5 \mu \mathrm{m}$. 
potential to distinguish between M1 and M2 phenotypes in terms of their difference in the iNOS level and thus the NO level. ${ }^{5-7}$ To this end, we set up a model of human macrophage polarization according to a previously reported method. ${ }^{3}$ Briefly, human monocytic THP-1 cells were first differentiated into macrophages by $24 \mathrm{~h}$ incubation with phorbol 12-myristate 13acetate (PMA) followed by $24 \mathrm{~h}$ incubation in RPMI medium; then, the macrophages were polarized in M1 macrophages by incubation with IFN- $\gamma / \mathrm{LPS}$, and in M2 macrophages by incubation with interleukin 4 (IL-4) and interleukin 13 (IL-13) (Fig. 5A). After a thorough wash to remove all stimuli, the M1and M2-polarized macrophages were treated with $\mathbf{1}$ (as a representative) and then imaged under a confocal laser scanning microscope. As shown in Fig. 5B, a bright intracellular green fluorescence could be observed in 1-loaded M1 macrophages, but not in 1-loaded M2 macrophages; moreover, when M1 macrophages were pretreated with NO synthase inhibitor AG and then treated with $\mathbf{1}$, the intracellular green fluorescence was greatly inhibited. The results indicate that 1 could discriminate M1 macrophages from M2 macrophages in terms of their difference in the iNOS level and thus the NO level. Of note, when M2 macrophages were pretreated with IFN- $\gamma /$ LPS and then treated with 1, the intracellular green fluorescence was greatly recovered, consistent with the report that M2 macrophages could be repolarized to M1 macrophages by IFN- $\gamma / \mathrm{LPS}$ treatment., ${ }^{9,10}$

Finally, we tested the ability of $\mathbf{1}$ to image NO communication during the phagocytosis of cancer cells by macrophages in a co-culture system containing M1- or M2-polarized macrophages and SKOV-3 human ovarian cancer cells. Briefly, the M1or M2-polarized macrophages were first stained with commercial blue-fluorescent nucleus dye DAPI, and then co-cultured

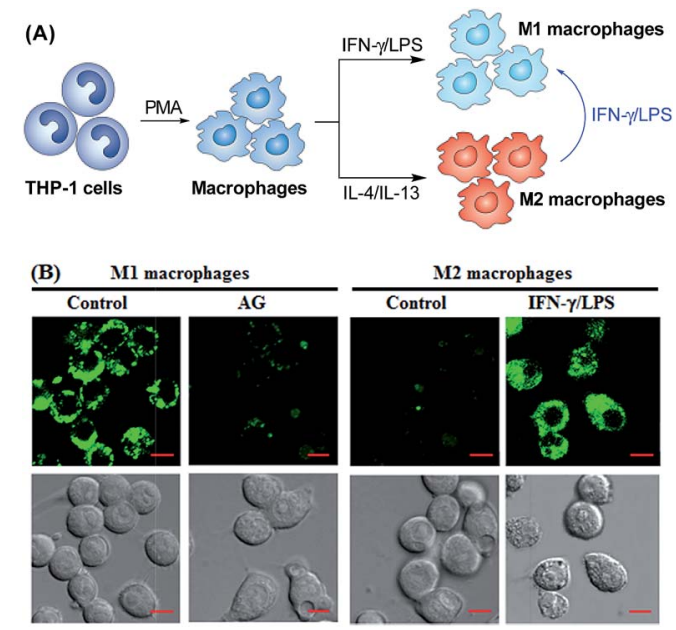

Fig. 5 (A) Macrophages can be differentiated starting from the human monocytic cell line THP-1. Once differentiated in the presence of PMA, they can be polarized into $M 1$ and $M 2$ macrophages by IFN- $\gamma / L P S$ and IL-4/IL-13 treatment, respectively. M1 macrophages could also be produced by the polarization of M2 macrophages by IFN- $\gamma /$ LPS. (B) Confocal fluorescence images of $\mathrm{M} 1$ or $\mathrm{M} 2$ macrophages treated with 1 in the absence and presence of AG or IFN- $\gamma /$ LPS. Emission was collected at $493-600 \mathrm{~nm}\left(\lambda_{\mathrm{ex}}=488 \mathrm{~nm}\right)$. Scale bar: $10 \mu \mathrm{m}$. with 1-loaded SKOV-3 cells for $12 \mathrm{~h}$, followed by imaging under a confocal laser scanning microscope. Note that the use of DAPI dye is in order to distinguish M1 or M2 macrophages from SKOV-3 cells in the co-culture system. As shown in Fig. 6A, the SKOV-3 cells cultured alone displayed almost no intracellular fluorescence in the green channel when treated with $\mathbf{1}$, indicating that the cancer cells express negligible intracellular NO. Upon capture by M1 macrophages in the co-culture system, the 1-loaded SKOV-3 cells exhibited a strong intracellular green fluorescence (Fig. 6B), indicating that during the immunemediated phagocytosis of cancer cells, NO secreted by M1 macrophages could diffuse across the cell membrane of cancer cells to exert its tumoricidal influence by producing cytotoxic $\mathrm{N}_{2} \mathrm{O}_{3}$ and $\mathrm{ONOO}^{-}$. The result was further supported by an inhibition assay, where the 1-loaded SKOV-3 cells captured by the AG-pretreated M1 macrophages displayed an obviously decreased intracellular green fluorescence (Fig. 6C). In sharp contrast, when the 1-loaded SKOV-3 cells were captured by M2 macrophages in the co-culture system, almost no intracellular green fluorescence could be observed in the former (Fig. 6D), in line with the low iNOS level in M2 macrophages. However, when M2 macrophages were pretreated with IFN- $\gamma /$ LPS and then

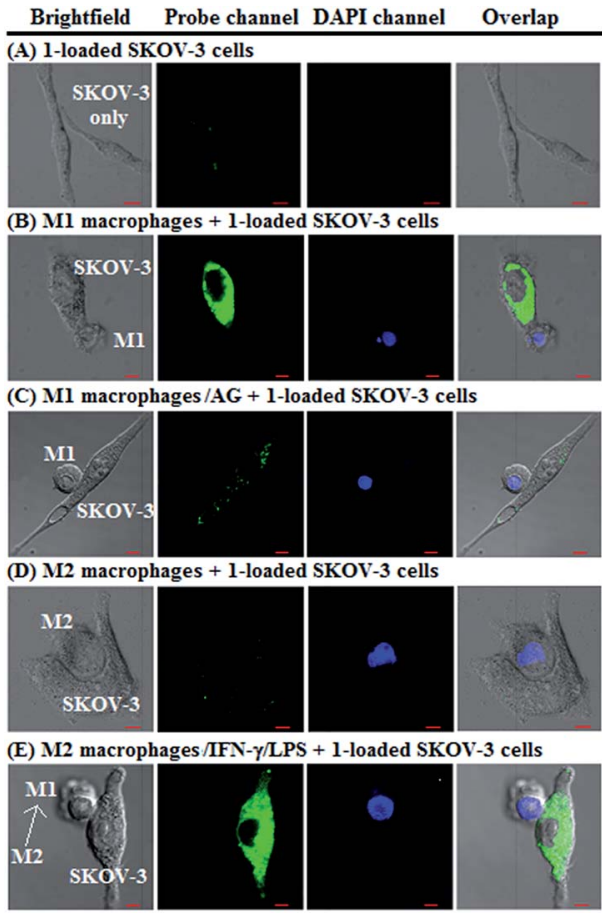

Fig. 6 Imaging NO communication between DAPI-stained macrophages and 1-loaded SKOV-3 cancer cells in a co-culture system. (A) 1-loaded SKOV-3 cancer cells only (as a control); (B) 1-loaded SKOV-3 cells captured by DAPI-stained M1 macrophages in the co-culture system; (C) 1-loaded SKOV-3 cells captured by DAPI-stained and AGpretreated M1 macrophages in the co-culture system; (D) 1-loaded SKOV-3 cells captured by DAPI-stained M2 macrophages in the coculture system; (E) 1-loaded SKOV-3 cells captured by DAPI-stained and IFN- $\gamma /$ LPS-pretreated M2 macrophages in the co-culture system. For the probe channel, emission was collected at $493-600 \mathrm{~nm}\left(\lambda_{\text {ex }}=\right.$ $488 \mathrm{~nm}$ ); for the DAPI channel, emission was collected at $410-485 \mathrm{~nm}$ $\left(\lambda_{\mathrm{ex}}=405 \mathrm{~nm}\right)$. Scale bar: $5 \mu \mathrm{m}$. 
co-cultured with 1-loaded SKOV-3 cells, the captured SKOV-3 cells displayed a dramatically increased intracellular green fluorescence (Fig. 6E), confirming that M2 macrophages can be polarized to M1 macrophages by IFN- $\gamma /$ LPS. ${ }^{9,10}$

Overall, the above results confirm that 1 could be used to discriminate M1 macrophages from M2 macrophages, evaluate the repolarization of TAMs from pro-tumoral M2 phenotype to anti-tumoral M1 phenotype, and image NO communication between macrophages and cancer cells during the immunemediated phagocytosis process, thus being very promising for imaging applications in cancer immunotherapy studies and relevant anti-cancer drug screening.

\section{Conclusions}

In summary, we in this work presented an aromatic secondary amine-functionalized Bodipy dye $\mathbf{1}$ and its mitochondriatargetable derivative Mito1 as highly sensitive fluorescent NO probes for discriminating M1 macrophages from M2 macrophages in terms of their difference in the iNOS level and thus the NO level. The high sensitivity of the two probes for NO originates from their unique ability to simultaneously respond to $\mathrm{N}_{2} \mathrm{O}_{3}$ and $\mathrm{ONOO}^{-}$. In this regard, the two probes should be superior to most of the existing fluorescent NO probes that can only respond to $\mathrm{N}_{2} \mathrm{O}_{3}$. Although an o-diamine-locked rhodamine lactam derivative has been reported to be able to give a similar fluorescence off-on response for both $\mathrm{N}_{2} \mathrm{O}_{3}$ and $\mathrm{ONOO}^{-}{ }^{38}$ the later studies found that this type of probe could suffer from serious interference by intracellular abundant Cys to lead to decreased sensitivity for NO. ${ }^{56}$ Importantly, with $\mathbf{1}$ as a representative, we have successfully realized the discrimination of M1 macrophages from M2 macrophages, evaluation of the repolarization of TAMs from pro-tumor M2 phenotype to anti-tumor M1 phenotype, and visualization of NO communication during the immune-mediated phagocytosis of cancer cells by M1 macrophages. Thus, our probes should hold great potential for NO-related physiological and pathological studies as well as anticancer drug screening in cancer immunotherapy.

\section{Conflicts of interest}

There are no conflicts to declare.

\section{Acknowledgements}

This work was supported by the Natural Science Foundation of China (No. 21877077, 21572121, 21778036, and 21502108), Natural Science Foundation for youth of Shanxi (No. 201601D021055), and Scientific and Technological Innovation Programs of Higher Education Institutions in Shanxi.

\section{Notes and references}

$1 \mathrm{~J}$. A. Ross and M. J. Auger, The biology of the macrophage, in The Macrophage, B. Burke and C. E. Lewis, Oxford University Press, Oxford, UK, 2nd edn, 2002.
2 R. Ostuni, F. Kratochvill, P. J. Murray and G. Natoli, Trends Immunol., 2015, 36, 229-239.

3 M. Genin, F. Clement, A. Fattaccioli, M. Raes and C. Michiels, BMC Cancer, 2015, 15, 577-590.

4 S. K. Biswas, P. Allavena and A. Mantovani, Semin. Immunopathol., 2013, 35, 585-600.

5 S. Edin, M. L. Wikberg, A. M. Dahlin, J. Rutegård, Å. Öberg, P. Oldenborg and R. Palmqvist, PLoS One, 2012, 7, e47045.

6 L. K. Keefer, W. J. Murphy, C. C. Harris, D. A. Wink and R. H. Wiltrout, J. Exp. Med., 2010, 207, 2455-2467.

7 S. A. Almatroodi, C. F. McDonald, I. A. Darby and D. S. Pouniotis, Cancer Microenviron., 2016, 9, 1-11.

8 E. Overmeire, D. Laoui, J. Keirsse, J. V. Ginderachter and A. Sarukhan, Front. Immunol., 2014, 5, 127.

9 D. Duluc, M. Corvaisier, S. Blanchard, L. Catala, P. Descamps, E. Gamelin, S. Ponsoda, Y. Delneste, M. Hebbar and P. Jeannin, Int. J. Cancer, 2009, 125, 367373.

10 M. D. Palma, R. Mazzieri, L. S. Politi, F. Pucci, E. Zonari, G. Sitia, S. Mazzoleni, D. Moi, M. A. Venneri, S. Indraccolo, A. Falini, L. G. Guidotti, R. Galli and L. Naldini, Cancer Cell, 2008, 14, 299-311.

11 C. M. Ohri, A. Shikotra, R. H. Green, D. A. Waller and P. Bradding, Eur. Respir. J., 2009, 33, 118-126.

12 A. Mantovani and P. Allavena, J. Exp. Med., 2015, 212, 435445.

13 N. N. Parayath, A. Parikh and M. M. Amiji, Nano Lett., 2018, 18, 3571-3579.

14 Y. Zhang, L. Wu, Z. Li, W. Zhang, F. Luo, Y. Chu and G. Chen, Biomacromolecules, 2018, 19, 2098-2108.

15 X. Ai, M. Hu, Z. Wang, L. Lyu, W. Zhang, J. Li, H. Yang, J. Lin and B. Xing, Bioconjugate Chem., 2018, 29, 928-938.

16 S. Zanganeh, G. Hutter, R. Spitler, O. Lenkov, M. Mahmoudi, A. Shaw, J. S. Pajarinen, H. Nejadnik, S. Goodman, M. Moseley, L. M. Coussens and H. E. Daldrup-Link, Nat. Nanotechnol., 2016, 11, 986-994.

17 M. Song, T. Liu, C. Shi, X. Zhang and X. Chen, ACS Nano, 2016, 10, 633-647.

18 X. Jiao, Y. Li, J. Niu, X. Xie, X. Wang and B. Tang, Anal. Chem., 2018, 90, 533-555.

19 M. Gao, F. Yu, C. Lv, J. Choo and L. Chen, Chem. Soc. Rev., 2017, 46, 2237-2271.

20 M. H. Lim and S. J. Lippard, J. Am. Chem. Soc., 2005, 127, 12170-12171.

21 L. E. McQuade, J. Ma, G. Lowe, A. Ghatpande, A. Gelperin and S. J. Lippard, Proc. Natl. Acad. Sci. U. S. A., 2010, 107, 8525-8530.

22 G. Sivaraman, T. Anand and D. Chellappa, ChemPlusChem, 2014, 79, 1761-1766.

23 H. Kojima, N. Nakatsubo, K. Kikuchi, S. Kawahara, Y. Kirino, H. Nagoshi, Y. Hirata and T. Nagano, Anal. Chem., 1998, 70, 2446-2453.

24 H. Kojima, Y. Urano, K. Kikuchi, T. Higuchi, Y. Hirata and T. Nagano, Angew. Chem., Int. Ed., 1999, 38, 3209-3212.

25 H. Kojima, M. Hirotani, N. Nakatsubo, K. Kikuchi, Y. Urano, T. Higuchi, Y. Hirata and T. Nagano, Anal. Chem., 2001, 73, 1967-1973. 
26 Y. Gabe, Y. Urano, K. Kikuchi, H. Kojima and T. Nagano, J. Am. Chem. Soc., 2005, 126, 3357-3367.

27 E. Sasaki, H. Kojima, H. Nishimatsu, Y. Urano, K. Kikuchi, Y. Hirata and T. Nagano, J. Am. Chem. Soc., 2005, 127, 3864-3865.

28 I. Johnson and M. T. Z. Spence, The Molecular Probes Handbook: a Guide to Fluorescent Probes and Labeling Technologies, Life Technologies, Carlsbad, Calif, USA, 11th edn, 2010.

29 L. A. Ridnour, D. D. Thomas, D. Mancardi, M. G. Espey, K. M. Miranda, N. Paolocci, M. Feelisch, J. Fukuto and D. A. Wink, Biol. Chem., 2004, 385, 1-10.

30 X. Zhang, W.-S. Kim, N. Hatcher, K. Potgieter, L. L. Moroz, R. Gillette and J. V. Sweedler, J. Biol. Chem., 2002, 277, 48472-48478.

31 X. Ye, S. S. Rubakhin and J. V. Sweedler, J. Neurosci. Methods, 2008, 168, 373-382.

32 T. Wang, E. F. Douglass Jr., K. J. Fitzgerald and D. A. Spiegel, J. Am. Chem. Soc., 2013, 135, 12429-12433.

33 S.-T. Wang, Y. Lin, C. D. Spicer and M. M. Stevens, Chem. Commun., 2015, 51, 11026-11029.

34 Y. Yang, S. K. Seidlits, M. M. Adams, V. M. Lynch, C. E. Schmidt, E. V. Anslyn and J. B. Shear, J. Am. Chem. Soc., 2010, 132, 13114-13116.

35 C.-G. Dai, J.-L. Wang, Y.-L. Fu, H.-P. Zhou and Q.-H. Song, Anal. Chem., 2017, 89, 10511-10519.

36 T.-W. Shiue, Y.-H. Chen, C.-M. Wu, G. Singh, H.-Y. Chen, C.-H. Hung, W.-F. Liaw and Y.-M. Wang, Inorg. Chem., 2012, 51, 5400-5408.

37 Y. Huo, J. Miao, Y. Li, Y. Shi, H. Shi and W. Guo, J. Mater. Chem. B, 2017, 5, 2483-2490.

38 H. Zheng, G.-Q. Shang, S.-Y. Yang, X. Gao and J.-G. Xu, Org. Lett., 2008, 10, 2357-2360.

39 Y.-Q. Sun, J. Liu, H. Zhang, Y. Huo, X. Lv, Y. Shi and W. Guo, J. Am. Chem. Soc., 2014, 136, 12520-12523.
40 C. Wang, X. Song, Z. Han, X. Li, Y. Xu and Y. Xiao, ACS Chem. Biol., 2016, 11, 2033-2040.

41 X. Zhang, B. Wang, Y. Xiao, C. Wang and L. He, Analyst, 2018, 143, 4180-4188.

42 S. Ma, D.-C. Fang, B. Ning, M. Li, L. He and B. Gong, Chem. Commun., 2014, 50, 6475-6478.

43 H. Li, D. Zhang, M. Gao, L. Huang, L. Tang, Z. Li, X. Chen and X. Zhang, Chem. Sci., 2017, 8, 2199-2203.

44 J. Miao, Y. Huo, X. Lv, Z. Li, H. Cao, H. Shi, Y. Shi and W. Guo, Biomaterials, 2016, 78, 11-19.

45 Z. Mao, H. Jiang, X. Song, W. Hu and Z. Liu, Anal. Chem., 2017, 89, 9620-9624.

46 Z. Mao, H. Jiang, Z. Li, C. Zhong, W. Zhang and Z. Liu, Chem. Sci., 2017, 8, 4533-4538.

47 C. J. Reinhardt, E. Y. Zhou, M. D. Jorgensen, G. Partipilo and J. Chan, J. Am. Chem. Soc., 2018, 140, 1011-1018.

48 R. Radi, J. Biol. Chem., 2013, 288, 26464-26472.

49 N. Nalwaya and W. M. Deen, Chem. Res. Toxicol., 2005, 18, 486-493.

50 G. Masanta, C. S. Lim, H. J. Kim, J. H. Han, H. M. Kim and B. R. Cho, J. Am. Chem. Soc., 2011, 133, 5698-5700.

51 A. R. Lippert, G. C. Van de Bittner and C. J. Chang, Acc. Chem. Res., 2011, 44, 793-804.

52 M. Masuda, H. F. Mower, B. Pignatelli, I. Celan, M. D. Friesen, H. Nishino and H. Ohshima, Chem. Res. Toxicol., 2000, 13, 301-308.

53 S. O. Raja, G. Sivaraman, A. Mukherjee, C. Duraisamy and A. Gulyani, ChemistrySelect, 2017, 2, 4609-4616.

54 R. B. Lorsbach, W. J. Murphy, C. J. Lowenstein, S. H. Snyder and S. W. Russell, J. Biol. Chem., 1993, 268, 1908-1913.

55 M. Shirhan, S. M. Moochhala, S. Y. Kerwin, K. C. Ng and J. Lu, Resuscitation, 2004, 61, 221-229.

56 Y. Huo, J. Miao, L. Han, Y. Li, Z. Li, Y. Shi and W. Guo, Chem. Sci., 2017, 8, 6857-6864. 